\author{
United Nations Educational Scientific and Cultural Organization \\ and \\ International Atomic Energy Agency
}

THE ABDUS SALAM INTERNATIONAL CENTRE FOR THEORETICAL PHYSICS

\title{
NON-LOCAL ENERGY DENSITY FUNCTIONALS: MODELS PLUS SOME EXACT GENERAL RESULTS
}

\author{
N.H. March \\ Oxford University, Oxford, England, \\ Department of Physics, University of Antwerp (RUCA), Antwerp, Belgium \\ and \\ The Abdus Salam International Centre for Theoretical Physics, Trieste, Italy.
}

\begin{abstract}
Holas and March (Phys. Rev. A51, 2040, 1995) gave a formally exact expression for the force $-\frac{\partial V_{x c}(\underset{\sim}{r})}{\partial \underset{\sim}{r}}$ associated with the exchange-correlation potential $V_{x c}(\underset{\sim}{r})$ of density functional theory. This forged a precise link between first- and second-order density matrices and $V_{x c}(\underset{\sim}{r})$. Here models are presented in which these low-order matrices can be related to the ground-state electron density. This allows non-local energy density functionals to be constructed within the framework of such models. Finally, results emerging from these models have led to the derivation of some exact 'nuclear cusp' relations for exchange and correlation energy densities in molecules, clusters and condensed phases.
\end{abstract}

MIRAMARE - TRIESTE

February 2001 


\section{Background and outline}

In their early attempts to transcend the Thomas-Fermi (TF) approximation, March and Young [MY:1] recognized that electron density theory should be regarded as a branch of density matrix theory. Their early studies led to a single-particle kinetic energy functional $T_{s}[\rho]$ of the form

$$
T_{s}[\rho]=T_{W}[\rho]+\Gamma(N) T_{T F}[\rho]
$$

where $T_{T F}[\rho]$ and $T_{W}[\rho]$ were the TF kinetic energy and the von Weizsäcker inhomogeneity kinetic energy respectively. In particular, the formula of MY for $\Gamma(N)$ led to this TF contribution correctly vanishing in the case of a single level occupancy only. Subsequently Herring and coworkers $[2,3]$ gave stronger arguments as to why in $T_{s}[\rho]$ it was physically appropriate to subtract out the full von Weizsäcker contribution $T_{W}[\rho]$ in constructing the single-particle kinetic energy functional $T_{s}[\rho]$.

Of course, the most common route to implement DFT has been since Slater's work [4] to return to orbitals from which to obtain the single-particle kinetic energy by standard quantum mechanics. The only question remaining then concerns the one-body potential $V(\underset{\sim}{r})$, now including exchange and correlation interactions, to insert into the Schrödinger equation (the so-called Slater-Kohn-Sham (SKS) equations [4,5]). However, within the last decade, major effort has been focussed on orbital free DFT [6-10]. This search for (presently approximations to) the single-particle kinetic energy functional $T_{s}[\rho]$ is then, of course, additional to the construction of $V(\underset{\sim}{r})$, given formally by

$$
V(\underset{\sim}{r})=V_{\text {Hartree }}(\underset{\sim}{r})+V_{x c}(\underset{\sim}{r})
$$

One direction of progress on the force $-\partial V_{x c} / \partial r_{\sim}^{r}$ associated with $V_{x c}(\underset{\sim}{r})$ was pointed out by Holas and March [11,12], who showed, via a many-electron form of the differential virial theorem [13] that the force $-\partial V_{x c}(\underset{\sim}{r}) / \partial \underset{\sim}{r}$ could be written, quite explicitly, in terms of first- and second-order density matrices (1 and 2DMs) of the many-electron system under discussion, plus, of course, information contained in the Slater-Kohn-Sham determinant of the 'reference' system with the same ground-state density as the correlated electron assembly under discussion. The problem of finding $V_{x c}(\underset{\sim}{r})$ is central, whether one works with SKS orbitals to obtain $T_{s}$, or with the single-particle kinetic energy functional $T_{s}[\rho]$ in orbital-free theory since the Euler-equation of DFT reads [14]

$$
\mu=\frac{\delta T_{s}}{\delta \rho(\underset{\sim}{r})}+V_{\text {Hartree }}(\underset{\sim}{r})+V_{x c}(\underset{\sim}{r})
$$

where $\mu$ is the chemical potential (constant throughout the entire charge cloud) of the molecule, cluster or condensed phase being considered.

The outline of the present work is then as follows. In section 2 below, a one-dimensional model of harmonic confinement of independent fermions will be utilized to expose a non-local 
form of the single-particle kinetic energy functional $T_{s}[\rho]$ which is exact in this model [15]. Though correlation kinetic energy is 'moved' into the exchange-correlation potential $V_{x c}(\underset{\sim}{r})$ in writing the chemical potential eqn.(1.3), it will be convenient for presentation to close section 2 with a short discussion of an explicit correlation kinetic energy functional, $T_{c}[\rho]$ say, for 2electron $\mathrm{He}$-like atomic ions in the nonrelativistic limit of large atomic number $Z$. This use of the so-called $1 / Z$ expansion in atomic theory, related to the TF statistical theory explicitly by March and White [16] leads naturally into section 3, where the focus is on the large $Z$ limit of atomic ions in which both $K$ and $L$ shells are now closed: i.e., one has moved from $\mathrm{He}$-like ions in section 2 to $N e$-like systems. In the limit of large atomic number $Z$, the exchange energy density $\epsilon_{x}(r)$ (defined precisely in eqn.(3.5) has been calculated exactly in the very recent work of Howard et al. [17]. A nuclear cusp condition is already exposed by the result for $\epsilon_{x}(r)$ in this limit system. Section 4 is devoted to the demonstration that this limiting result for $\epsilon_{x}$ is exactly true, not only in atomic ions of arbitrary atomic number but also for multicentre problems of molecular and solid state theory [18]. The same nuclear cusp condition is shown to embrace the correlation energy, also in section 4 . After a brief summary in section 5, some proposals are made there for future studies.

\section{Non-local kinetic energy density functional}

In this section, some recent results on a model in which a non-local kinetic energy functional can be obtained will first be summarized. However, this is a model of independent Fermions, so that it is only relevant to the single-particle kinetic energy functional $T_{s}[\rho]$. At the end of this section, some results will also be reported on correlation kinetic energy, but now in the restricted case of He-like ions having large atomic number $Z$. Throughout the paper, the considerations are restricted to non-relativistic quantum mechanics.

\subsection{Arbitrary number of Fermions in a one-dimensional harmonic oscillator potential}

In early work, Lawes and March [19] studied the admittedly very specialized model of $N$ independent Fermions filling singly the levels of the linear harmonic oscillator potential $V(x)=\frac{1}{2} x^{2}$. For this model, they already achieved a long-term aim of density functional theory (DFT) in that they derived an exact differential equation for the ground-state density $\rho(x)$ : namely

$$
\frac{\rho^{\prime \prime \prime}(x)}{8}+\frac{1}{2} V^{\prime}(x) \rho(x)+(N-V(x)) \rho^{\prime}(x)=0
$$

The lowest level only being occupied here corresponds to putting $N=1$ in eqn.(2.1).

Still in one dimension, March and Young [13] had earlier derived the so-called differential virial theorem. Defining the (single-particle) kinetic energy per unit length $t_{s}(x)$ from the one- 
body wave functions $\psi_{i}(x)$ generated by the potential energy $V(x)$ as

$$
t_{s}(x)=-\frac{1}{2} \sum_{i=1}^{N} \psi_{i}^{*}(x) \frac{\partial^{2} \psi_{i}(x)}{\partial x^{2}}
$$

these authors [13] showed, but now for a general potential $V(x)$ which allowed normalization of $\rho(x)$ to $N$ particles, that

$$
t_{s}^{\prime}(x)=-\frac{1}{2} \rho V^{\prime}(x)-\frac{\rho^{\prime \prime \prime}(x)}{8}
$$

Applying this general one-dimensional result (2.3) to the above model of harmonic confinement, one can immediately rewrite eqn.(2.1) as [15]

$$
\frac{t_{s}^{\prime}(x)}{\rho^{\prime}(x)}=\left(N-\frac{1}{2} x^{2}\right)
$$

Differentiating eqn.(2.4) with respect to $x$ yields

$$
\left[\frac{t_{s}^{\prime}(x)}{\rho^{\prime}(x)}\right]^{1}=-x \equiv-\frac{\partial V}{\partial x}
$$

and substituting this harmonic oscillator result for $\partial V / \partial x$ into eqn.(2.3) gives

$$
t_{s}^{\prime}(x)=+\frac{1}{2} \rho\left[\frac{t_{s}^{\prime}(x)}{\rho^{\prime}(x)}\right]^{1}-\frac{1}{8} \rho^{\prime \prime \prime}(x)
$$

In ref.[15], eqn.(2.6) is integrated to obtain, after some manipulation

$$
t_{s}(x)=-\frac{\rho^{\prime \prime}(x)}{12}+\left[\frac{N}{3 \rho^{2}(0)}+\frac{1}{12} \int_{0}^{x} d s \frac{\rho^{\prime \prime \prime}(s)}{\rho^{2}(s)}\right] \rho^{3}(x) .^{*}
$$

Integrating further, eqn.(2.7) can be expressed as

$$
t_{s}(x)=t_{W}(x)+\xi(x) t_{T F}(x)
$$

where the Thomas-Fermi (TF) and von Weizsäcker (W) kinetic energy densities (see also eqn.(1.1)) are defined in one dimension as

$$
t_{T F}(x)=c_{k} \rho^{3}(x): c_{k}=\pi^{2} / 24
$$

and

$$
t_{W}(x)=\frac{1}{8} \frac{\rho^{\prime 2}(x)}{\rho(x)} .
$$

What emerges is that, while the form (2.8) is not, of itself, new [20-22], $\xi(x)$ in this model can be obtained exactly as

$$
\xi(x)=\xi(0)+\frac{4}{3} \int_{0}^{x} d x\left[\frac{t_{W}^{\prime}(x)}{t_{T F}(x)}-\left(\frac{t_{W}(x)}{t_{T F}(x)}\right)^{\prime}\right]
$$

*Note added after completion of MS. This result (2.7) has now been extended to three-dimensional isotropic harmonic confinement by A. Minguzzi, N.H. March and M.P. Tosi (to be published). 
Thus $\xi(x)-\xi(0)$ can itself be expressed solely in terms of $t_{T F}(x)$ and $t_{W}(x)$ as building blocks. The quantity $\xi(0)$ is evidently from eq.(2.8) given by

$$
\xi(0)=\frac{t_{s}(0)-t_{W}(0)}{t_{T F}(0)}
$$

and $t_{s}(0)$ from eqn.(2.7) has the value

$$
t_{s}(0)=\frac{N \rho(0)}{3}-\frac{\rho^{\prime \prime}(0)}{12}
$$

Hence $\xi(0)$ itself is known in terms of $\rho, \rho^{\prime}$ and $\rho^{\prime \prime}$, all evaluated at $x=0$, using eqns.(2.9) and (2.10) and the number of Fermions $N$ given by

$$
N=\int_{-\infty}^{\infty} \rho(x) d x
$$

Thus, with complete knowledge of $\xi(x)$ in terms of $\rho(x)$ and its low-order derivatives in this model, the single-particle density is obtained in a fully non-local form via eqns.(2.8), (2.11) and (2.12)-(2.14). Evidently, again of course for this model, the (single-particle) kinetic energy functional $T_{s}$ follows from eqn.(2.8) as [15]

$$
\begin{aligned}
T_{s} & =\int_{-\infty}^{\infty} t_{s}(x) d x \\
& =\int_{-\infty}^{\infty} \frac{1}{8} \frac{\rho^{\prime}(x)^{2}}{\rho} d x+\int_{-\infty}^{\infty} \xi(x) t_{T F}(x) d x \\
& \equiv T_{W}+\int_{-\infty}^{\infty} \xi(x) t_{T F}(x) d x
\end{aligned}
$$

This then is the non-local kinetic energy functional in this model of harmonic confinement in one dimension.

A byproduct of the above argument [15] is the relation

$$
\frac{t_{s}^{\prime}(x)}{\rho^{\prime}(x)}=\frac{3 t_{s}(x)}{\rho(x)}+\frac{\rho^{\prime \prime}(x)}{4 \rho(x)}
$$

and then it follows that

$$
\frac{\delta T_{s}}{\delta \rho(x)}=\frac{3 t_{s}(x)}{\rho(x)}+\frac{\rho^{\prime \prime}(x)}{4 \rho(x)}+C(N)
$$

where $C$ is a constant, depending however on the number of Fermions $N$. It is, of course, this functional derivative which is needed to solve the Euler eqn.(1.3) of DFT.

To close this section on the kinetic energy functional, we turn to the explicit example of two (antiparallel) spin electrons repelling Coulombically.

\subsection{Correlation effects with two (antiparallel) spin electrons repelling Coulom- bically}

It is the aim of this section to treat two cases: 
(i) When the iteracting electrons are again harmonically confined - the so-called Hookean atom going back to Kestner and Sinanoglu [23, 24]

and

(ii) Helium-like ions in the limit of large $Z$.

Since the differential eqn.(2.1) of Lawes and March for (non-interacting) harmonic confinement was a key result in obtaining the single-particle kinetic energy functional (2.15), we note that in case (i) above the study of March, Gál and Nagy [25] yields the corresponding equation, now for two electrons only but incorporating Coulomb repulsion exactly: for force constant $k=1 / 4$ :

$$
\begin{array}{r}
\left(-r^{2}+r^{4}\right) \rho^{\prime \prime \prime}(r)+\left(-2 r-4 r^{3}+4 r^{5}\right) \rho^{\prime \prime}(r) \\
+\left(2-13 r^{2}-4 r^{4}+5 r^{6}\right) \rho^{\prime}(r)+\left(-10 r^{3}+2 r^{2}\right) \rho(r)=0
\end{array}
$$

There is substantial similarity between eqn.(2.18) for two electrons and eqn.(2.1) for an arbitrary number of harmonically confined electrons. In particular, both are homogeneous linear thirdorder differential equations.

In view of the work of Holas and March [11] on the exchange-correlation potential in relation to 1 and 2DMs, it is noteworthy that the same Hookean atom model with $k=\frac{1}{4}$ also relates 1 and $2 \mathrm{DMs}$ through [26]

$$
\begin{aligned}
& \left(1+\frac{1}{2}\left|\underset{\sim}{r}-{\underset{\sim}{r}}^{\prime}\right|\right) \gamma\left(\underset{\sim}{r}, r_{\sim}^{\prime}\right)=\left\{n_{2}\left(\underset{\sim}{r}, r_{\sim}^{\prime}\right)\right\}^{1 / 2} \int d{\underset{\sim}{1} 1}_{1}\left[1+\frac{1}{2}\left|\underset{\sim}{r}-\underset{\sim_{1}}{r}\right|\right]\left[1+\frac{1}{2}\left|\sim_{1}-{\underset{\sim}{r}}^{\prime}\right|\right] \\
& \left\{n_{2}\left(\underset{\sim_{1}}{r}, \underset{r_{1}}{r}\right)\right\}^{1 / 2}
\end{aligned}
$$

Here $\gamma^{2}<\gamma$ because of the full treatment of interactions, and hence this 1DM (2.19) includes correlation kinetic energy. The pair function $n_{2}\left(r, r_{\sim}^{\prime}\right)$ is the diagonal element of the 2DM. Having focussed on two of the principle analytic results involving antiparallel spin electron correlation, namely the differential eqn.(2.18) for $\rho(r)$ and the relation (2.19) between the 1DM and the (diagonal) pair function of the 2DM with harmonic confinement, let us turn to case (ii) introduced above.

\subsection{He-like atomic ions with large atomic number}

Gál, March and Nagy [27] have subsequently obtained a differential equation analogous to eqns.(2.1) and (2.18) for He-like atomic ions in the (non relativistic) limit of large atomic number $Z$. The ground-state density $\rho(r)$ again satisfies a differential equation which is linear, third-order and homogeneous, namely

$$
\sum_{i=0}^{3} P_{i}(r) \rho^{(i)}(r)=0
$$


where $\rho^{(i)}(r)$ denotes the $\mathrm{i}^{\text {th }}$ derivative of $\rho(r)$ : with $\rho^{(0)}(r) \equiv \rho(r)$. The quantities $P_{i}(r)$ are also polynomials as in the Hookean eqn.(2.18) and are recorded explicitly by Gál et al. [27].

Turning to the 1DM, $\gamma\left(\underset{\sim}{r}, r^{\prime}\right)$ the work of Hall, Jones and Rees [28] was used by Gál et al. [27] to write, in the limit (2-electron) system with large atomic number $Z$ :

$$
\gamma\left(\underset{\sim}{r}, r_{\sim}^{\prime}\right)=\frac{\rho\left(r^{\prime}\right)}{2}\left[\frac{\rho_{0}(r)}{\rho_{0}\left(r^{\prime}\right)}\right]^{1 / 2}+\frac{\rho(r)}{2}\left[\frac{\rho_{0}\left(r^{\prime}\right)}{\rho_{)}(r)}\right]^{1 / 2}
$$

where $\rho_{0}(r)$ is the bare Coulombic density given by

$$
\rho_{0}(r)=\frac{Z^{3}}{\pi} \exp (-2 Z r)
$$

This result for $\gamma$ is readily used to obtain the correlation kinetic energy $T_{c}[\rho]$ in this limit system (2 electrons: large $Z$ ) as

$$
T_{c}[\rho]=-T_{W}[\rho]-\frac{1}{2}\left[\frac{\rho^{\prime}(r)}{\rho(r)}\right]_{r=0} \int \frac{\rho(r)}{r} d r_{\sim}-\frac{1}{8}\left\{\left[\frac{\rho^{\prime}(r)}{\rho(r)}\right]_{r=0}\right\}^{2} \int \rho(r) d r_{\sim}
$$

where Kato's theorem (see eqn.(3.8) below) has been employed to remove the atomic number $Z$. Here $T_{W}[\rho]$ is the (now, of course, three-dimensional) von Weizsäcker inhomogeneity kinetic energy in eqn.(1.1): namely

$$
T_{W}[\rho]=\frac{1}{8} \int \frac{(\nabla \rho)^{2}}{\rho} d r
$$

This discussion of electron-electron interaction in 2-electron atomic ions in the limit of large $Z$ leads us naturally to a discussion of exchange in such a limit system, but now with $K$ plus $L$ closed shells, i.e. a 10-electron case.

\section{Exchange energy density: model of 10 electron atomic ions in the limit of large atomic number $Z$}

In early work, March and Santamaria [29] obtained the Dirac density matrix $\gamma\left(\underset{\sim}{r}, r_{\sim}^{\prime}\right)$ for a hydrogenic system with filled $K$ and $L$ shells. The shape of their result is that $\gamma$ is related to the diagonal density $\rho$, which is spherically symmetric in this closed shell example, by

$$
\gamma\left(\underset{\sim}{r}, r_{\sim}^{\prime}\right)=\rho\left(\frac{r+r^{\prime}}{2}\right)+\left|\underset{\sim}{r}-{\underset{\sim}{r}}^{\prime}\right|^{2} F\left(\frac{r+r^{\prime}}{2}\right)
$$

The function $F$ in turn is related to $\rho$ itself through

$$
F(r)=\frac{1}{24} \rho^{\prime \prime}(r)-\frac{2 m}{\hbar^{2}} t_{s}(r),
$$

since, as discussed in section 2 above, $t_{S}$ is itself determined by $\rho$. In this bare Coulomb field model, $F(r)$ has the compact form

$$
F(r)=-\frac{1}{64 \pi}\left[\frac{Z}{a_{0}}\right]^{5} \exp \left(-\frac{Z r}{a_{0}}\right): a_{0}=\frac{\hbar^{2}}{m e^{2}} .
$$


Going back to the study of Dirac [30] the exchange energy $E_{x}$ of a system with idempotent 1DM $\gamma$ is given by

$$
E_{x}=-\frac{1}{4} e^{2} \int \frac{\gamma^{2}\left(\underset{\sim}{r}, \sim_{\sim}^{\prime}\right)}{\left|\underset{\sim}{r}-r_{\sim}^{\prime}\right|} d r \underset{\sim}{r} d r^{\prime}
$$

and from this one can define an exchange energy density $\left.\epsilon_{x} \underset{\sim}{r}\right)$ which is everywhere less than zero as

$$
\epsilon_{x}(r)=-\frac{1}{4} e^{2} \int \frac{\gamma^{2}\left(\underset{\sim}{r}, \stackrel{\sim}{r}^{\prime}\right)}{\left|\underset{\sim}{r}-{\underset{\sim}{r}}^{\prime}\right|} d r_{\sim}^{\prime}
$$

In the study of Howard et al. [17], $\epsilon_{x}(r)$ has been obtained in the limit system defined by the bare Coulomb density matrix (3.1). As noted in ref.[17], this is the limit of large $Z$ in the spirit of the $1 / Z$ expansion [16] for 10-electron atomic ions. The quantity $\epsilon_{x}(r)$ is given in closed form, but we wish to note here mainly two analytical limits which flow from that analysis, before presenting results in graphical form.

First, as the present writer [18] showed elsewhere,

$$
\left.\epsilon_{x}(r)\right|_{r \rightarrow \infty} \longrightarrow-\frac{e^{2}}{2 r} \rho(r)
$$

and this is shown to be quickly useful away from the nucleus in reproducing the exact results in ref.[17].

Secondly, and more importantly in the present context, a direct plot of $\epsilon_{x}(r)$ vs $r$ shows that $\epsilon_{x}$ is coming away from the nucleus $(r=0)$ with a finite slope. This can be found from the analytical formula for $\epsilon_{x}(r)[17]$ and the slope is such that

$$
\frac{\left.\epsilon_{x}^{\prime}(r)\right|_{r=0}}{\left.\epsilon_{x}(r)\right|_{r=0}}=-\frac{2 Z}{a_{0}}
$$

which has analogy with Kato's theorem for the electron density in a closed shell atomic ion of nuclear charge $Z e$ :

$$
\left.\frac{\rho^{\prime}(r)}{\rho(r)}\right|_{r=0}=-\frac{2 Z}{a_{0}}
$$

While eqn.(3.7) is obtained, at this point, only for the bare Coulomb model density matrix given by eqns.(3.1) and (3.3), it will be discussed further for 'real' atoms, molecules and solids in section 4 below.

However, while Kato's result (3.8) is already applicable to 'real' closed shell atomic ions, we next note, in the present context, that for closed shells (of arbitrary number in fact) in the bare Coulomb limit the spatial generalization of eqn.(3.8) exists, namely

$$
\frac{\partial \rho(r)}{\partial r}=-\frac{2 Z}{a_{0}} \rho_{\ell=0}(r)
$$


i.e., the total density $\rho(r)$ can be derived by integration of eqn.(3.9) from the density of $s(\ell=0)$ electrons alone [32].

In the framework of the present paper, which is focussed on relating low order density matrix to the electron density $\rho$, it is also noteworthy that Theophilou and March [33] have shown that the diagonal relation between total and $s(\ell=0)$ densities in this bare Coulomb limit has a counterpart with density matrices. Their relation is in fact

$$
\gamma\left(\underset{\sim}{r}, r_{\sim}^{\prime}\right)=-(x-y)^{-1}\left[\frac{\partial}{\partial x}-\frac{\partial}{\partial y}\right]\left[x y \gamma_{\ell=0}\left(\frac{x}{2}, \frac{y}{2}\right)\right]
$$

where

$$
x=r+r^{\prime}+\left|\underset{\sim}{r}-\stackrel{\sim}{r}^{\prime}\right|, \quad y=r+r^{\prime}-\left|\underset{\sim}{r}-{\underset{\sim}{r}}^{\prime}\right|
$$

The variables $x$ and $y$ in eqn.(3.11) are in fact motivated by the extra constant of motion in the bare Coulomb limit system, namely the Runge-Lenz vector [34]. Again, as with eqn.(3.9), the result (3.10) is valid for an arbitrary number of closed shells in this model.

Eqns.(3.5) and (3.10) clearly imply that the exchange energy density $\epsilon_{x}(r)$ calculated in ref.[17] is determined solely by the $s$ electron density matrix $\gamma_{\ell=0}\left(r, r^{\prime}\right)$, even though it was in fact obtained by direct insertion of eqns.(3.1) and (3.2) into the definition (3.5).

Howard et al. [17] also discuss the exchange potential $\left.V_{x} \underset{\sim}{r}\right)$ defined by

$$
V_{x}(\underset{\sim}{r})=\frac{\delta E_{x}}{\delta \rho(r)}
$$

but because of the results in eqns.(3.1), (3.2) and (3.4), this still involves a functional derivative with respect to $\rho(r)$ of $t_{s}$; e.g. $\delta t_{s}(r) / \delta \rho\left(\sim_{\sim}^{\prime}\right)$ and presently this is not known in this model in analytical form. However, approximations to $V_{x}(\underset{\sim}{r})$ due to (a) Slater [35] and (b) Harbola-Sahni [36] were investigated numerically using the exact density in this Coulomb limit system, the Slater approximation $V_{x}^{S \ell}(\underset{\sim}{r})$ being particularly simple since

$$
\left.V_{x}^{S \ell} \underset{\sim}{r}\right)=\frac{2 \epsilon_{x}(\underset{\sim}{r})}{\rho(\underset{\sim}{r})}
$$

This, using eqn.(3.6) tends to $-e^{2} / r$ at large $r$, which is correct.

Returning to $\epsilon_{x}(r)$ itself, Howard et al. [17] plotted the 'radial' exchange energy density $4 \pi r^{2} \epsilon_{x}(r)$ versus $r$ in their work, and this is reproduced in Fig.3.1. On the same plot, the local density approximation (LDA):

$$
\epsilon_{x}^{L D A}(r)=-c_{x} \rho(\underset{\sim}{r})^{4 / 3}: c_{x}=\frac{3}{4} e^{2}\left(\frac{3}{\pi}\right)^{1 / 3}
$$

going back to Dirac [30] and utilized especially by Slater [4] is given, but we stress with the exact density $\rho(r)$ used. Even though the point-point agreement is remarkable, the total exchange energy $E_{x}$ is in error by some $8 \%$ when the exchange $\epsilon_{x}(r)$ is replaced by the LDA form (3.8). 
In closing this section on exchange, we note qualitatively similar results to the above for a quite different model, namely that of electron confinement by a metal surface. This has been discussed quite recently by the writer [37]. What we wish to stress here is that in the (infinite barrier) model of a surface, the 1DM $\gamma\left(\underset{\sim}{r}, r_{\sim}^{\prime}\right)$ can be directly related to its diagonal density. This then allows a direct non-local relation to be established between $\epsilon_{x}(\underset{\sim}{r})$ and the kinetic energy density $t_{s}(\underset{\sim}{r})[37]$.

\section{Some exact general results: nuclear cusp conditions on ex- change and correlation energy densities}

Sections 2 and 3 comprise exact density functional results from models. While such exactitude is important for DFT, 'universality' of the functionals obtained cannot be claimed. However, the purpose of this section is to demonstrate that the Coulomb model result (3.7) is not only true in 'real' closed shell atomic ions, but also in multicentre problems of molecules, clusters and condensed matter.

The detailed argument will be presented elsewhere [18]: here we shall restrict ourselves to (a) the principles underlying the derivation and (b) the exact results which flow from these.

\subsection{Underlying principles employed}

The singularity of the nucleus $A$, with charge $Z_{A} e$, of the electron-nucleus interaction $V_{A}(\underset{\sim}{r})=$ $-Z_{A} e^{2} /|\underset{\sim}{r}-\underset{\sim}{R}|$ results in non-analyticity of the $N$-electron wave function when each electronic

coordinate $\underset{\sim_{i}}{r}, i=1,2 \ldots N$ becomes equal to $\underset{\sim}{R}$, yet the function remains finite everywhere. The characterization of this analyticity was first revealed in the pioneeering work of Kato [38], eqn.(3.8) of the previous section being an example of his findings, for the case of a closed shell atomic ion. Bingel [39] extended the treatment to a molecule, but here the presentation used by Davidson [40] will provide the basic background needed to present the results relevant to non-local energy density functionals.

With $H$ the total Hamiltonian, let the $N$-electron antisymmetric normalized wave function $\Psi\left({\underset{\sim}{i}}_{i}-{\underset{\sim}{x}}_{N}\right)$ be an eigenfunction of the Schrödinger equation

$$
H \Psi=E \Psi
$$

where $\underset{\sim_{i}}{x} \equiv\left\{\sim_{i} \underset{i}{r}, s_{i}\right\}$ denotes the space and spin coordinates of the $\mathrm{i}^{\text {th }}$ electron. In treating cusp conditions due to the specified $A^{\text {th }}$ nucleus, let us choose its position to be at $\underset{\sim}{R}=0$. As $\Psi$ is everywhere finite, it follows from eqn.(4.1) if we focus on the argument $\underset{\sim_{1}}{r}$ of $\Psi$ that

$$
\lim _{r_{1} \rightarrow 0} r_{1} H \Psi=0
$$

This implies that $\Psi\left(\underset{\sim_{1}}{r}, s_{i}, \underset{\sim_{2}}{x} \ldots \underset{\sim_{N}}{x}\right)$ is also an eigenfunction, with zero eigenvalue of the operator 


$$
b\left(\sim_{1}\right)=\lim _{r_{1} \rightarrow 0} r_{1} T=\lim _{r_{1} \rightarrow 0} r_{1} t\left({\underset{\sim}{1}}_{1}\right)-Z_{A}
$$

where $T=\sum_{i=1}^{N} t\left({\underset{\sim}{i}}_{i}^{r}\right)$ is the kinetic energy operator. For the wave function $\Psi\left(\underset{\sim_{1}}{r}, \underset{\sim_{1}}{x}\right)$ where $\underset{\sim}{x} \equiv\left\{s_{i}, \underset{\sim_{2}}{x} \ldots \underset{\sim_{N}}{x}\right\}$ expanded in spherical harmonics:

$$
\Psi(\underset{\sim}{r}, \underset{\sim}{x})=\sum_{\ell=0}^{\infty} \sum_{m=-\ell}^{m=\ell} y_{e m}\left(\Omega_{1}\right) r_{1}^{\ell} f_{\ell m}\left(r_{1}, \underset{\sim}{x}\right)
$$

with $f_{\ell m}\left(r_{1}, x\right)$ an analytic function of $r$, the eigenequation $b\left(\sim_{1}\right) \Psi=0$ yields the following conditions on the partial wave amplitudes [40]

$$
\left.\frac{\partial f_{\ell m}\left(r_{1}, \underset{\sim}{x}\right)}{\partial r_{1}}\right|_{r_{1}=0}=-\frac{Z_{A}}{\ell+1} f_{\ell m}(0, \underset{\sim}{x}) .
$$

These eqns.(4.3)-(4.5) are used by March et al. [18] to obtain nuclear cusp conditions for the exchange and correlation energy densities of DFT.

Before turning to the DFT energy densities $\epsilon_{x} \underset{\sim}{(r)}$ (discussed in section 3 above for the case of a 10-electron closed shell atomic ion in the limit of large $Z$ ) and the corresponding correlation (c) energy density $\epsilon_{c}(\underset{\sim}{r})$, for DFT applications the classical electrostatic energy of electrons is of interest, namely

$$
E_{e s}[\rho]=\int d \tilde{\sim}_{1} \epsilon_{e s}\left({\underset{\sim}{1}}_{1}\right)
$$

where

$$
\epsilon_{e s}\left({\underset{\sim}{1}}_{1}\right)=\int d \tilde{\sim}_{2} \frac{e^{2}}{\left|\sim_{1}^{r}-{\underset{\sim}{2}}_{2}^{r}\right|} \frac{1}{2} \rho\left(\underset{\sim_{1}}{r}\right) \rho\left({\underset{\sim}{2}}_{2}^{r}\right)
$$

March et al. [18] show that

$$
\left.\frac{\partial \epsilon_{e s}(r)}{\partial r_{1}}\right|_{r_{1}=0}=-2 Z_{A} \epsilon_{e s}(0)
$$

where the bar on $\epsilon_{e s}\left({\underset{\sim}{1}}_{1}^{r}\right)$ denotes an angular average. This is a simple analogue, for the classical electrostatic energy density, of eqn.(3.7) for exchange $(x)$ energy density derived from the bare Coulomb limit system (for a 10-electron atomic ion). This leads us to a sketch of the generalization of eqn.(3.7) derived for a model closed shell atomic ion to the fully interacting electron problem for atoms, molecules and solids [18]. The derivation in ref.[18] appeals to the adiabatic connection, developed by Harris and Jones [41,42]. This connection links the fully interacting system treated above to the SKS noninteracting reference system with the same (i.e. formally exact) electron density via intermediate systems. For this purpose, a Hamiltonian depending on a parameter $\alpha$ such that $0 \leq \alpha \leq 1$, is introduced to write

$$
H^{\alpha}=T+V^{\alpha}+\alpha U
$$


where $V^{\alpha}=\sum_{i=1}^{N} V^{\alpha}(\underset{i}{r})$. This Hamiltonian for $\alpha=1$ reduces to the original Hamiltonian while for $\alpha=0$ the electron-electron piece $U$ is switched off, while $\left.V^{0} \underset{\sim}{r}\right)=V_{S K S}(\underset{\sim}{r})$.

To use the above approach to investigate the cusp conditions for the intermediate system, March et al. [18] assume (to be checked later) that the properties of $V^{\alpha}(\underset{\sim}{r})$, whose explicit form remains unknown, permit a finite value for the limit $\gamma^{\alpha}=\lim _{r_{1} \rightarrow 0} r_{1} V^{\alpha}\left(\underset{\sim_{1}}{r}\right)$. Then the early arguments carry through and one obtains

$$
\left.\frac{\partial}{\partial r} \bar{\rho}^{\alpha}(r)\right|_{r=0}=2 \gamma^{\alpha} \rho^{\alpha}(0)
$$

which is the analogue of eqn.(3.8). But the one-body potential $\left.V^{\alpha} \underset{\sim}{r}\right)$ is defined by requiring that the electron density $\left.\rho^{\alpha} \underset{\sim}{r}\right)$ of the intermediate system is independent of $\alpha$. Hence we conclude that $\gamma^{\alpha}=-Z_{A}$ which justifies a posteriori the assumption that $\lim _{r_{1} \rightarrow 0} r_{1} V^{\alpha}\left(r_{1}\right)$ is finite.

March et al. [18] show that for the exchange-correlation potential $V_{x c}(r)$

$$
\lim _{r_{1} \rightarrow 0} r_{1} V_{x c}\left(\sim_{1}^{r}\right)=0
$$

Without essentially new arguments beyond those presented above, the model result (3.7) for the exchange energy density $\epsilon_{x}$ generalizes to

$$
\left.\frac{\partial \overline{\epsilon_{x}\left(r_{1}\right)}}{\partial r_{1}}\right|_{r_{1}=0}=-2 Z_{A} \epsilon_{x}(0)
$$

where the angular average on the LHS is not required for closed shell atomic ions. The final exact result of March et al. [18] is an analogous nuclear cusp condition for correlation energy density $\epsilon_{c}(\underset{\sim}{r})$, namely

$$
\left.\frac{\partial \overline{\epsilon_{c}\left(r_{1}\right)}}{\partial r_{1}}\right|_{r_{1}=0}=-2 Z_{A} \epsilon_{c}(0)
$$

These exact results (4.12) and (4.13) should evidently be built into approximate DFT theory in the future.

\section{Summary}

The early connection between density matrix and density functional theories stressed by March and Young [1] (DFT was not used then, but electron density theory) in deriving the approximate single-particle kinetic energy functional (1.1) has been greatly strengthened, especially during the last decade. Examples are now available in which an idempotent 1DM can be expressed, either directly or via the single-particle kinetic energy, in terms of the diagonal electron density $[15,17,37]$. Correlation can be added in two-electron atomic ions at large atomic number, to allow the 1DM to embrace correlation kinetic energy [27]. 
As to exchange energy, an idempotent closed shell 10-electron 1DM for the Coulomb limit system allows explicit analytical calculation of the exchange energy density $\epsilon_{x}(r)$ and this has been compared with LDA (but using the exact Coulomb ground-state electron density) in Fig.1. While point-to-point agreement between the two is excellent over the range where $4 \pi r^{2} \epsilon_{x}(r)$ is substantial, there are errors in LDA both near the nucleus and at large $r$. In the latter case, the exact asymptotic form is known (compare eqn.(3.6)) while at small $r$ the nuclear cusp condition (3.7) emerges from the model. But in this case, the model result proves generalizable not only to 'real' atomic ions, but also to molecules, clusters and condensed phases [see ref.[18], which is briefly summarized in section 4 above]. An exact parallel for the correlation energy $\epsilon_{c}(\underset{\sim}{r})$ follows.

As to future directions, it remains of central interest to improve available forms of the exchange-correlation potential $V_{x c}(\underset{\sim}{r})$. While it has been known since the work of Kohn and Sham [5] that one formal definition is

$$
V_{x c}(\underset{\sim}{r})=\frac{\delta E_{x c}[\rho]}{\delta \rho(\underset{\sim}{r})}
$$

already approximate forms available for the exchange-correlation energy functional $E_{x c}(\rho)$ have their errors exacerbated by the functional differentiation in eqn.(5.1). Again it seems that one can look to the further synthesis of density matrix and density functional theory via the manyelectron force-balance equation derived by Holas and March [11], namely

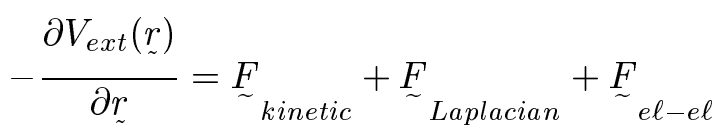

and a corresponding (one-body) equation for $V(\underset{\sim}{r})$ in eqn.(1.2) in terms of SKS orbitals, or non-interacting 1 and 2DMs built from a single determinant of these orbitals. In eqn.(5.2) also,

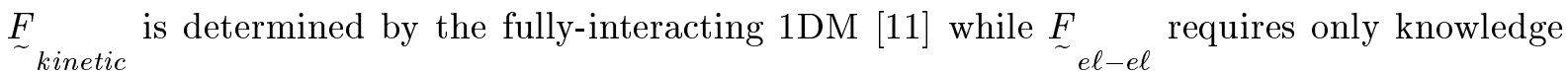
of the electronic pair correlation function $n_{2}\left(\underset{\sim}{r}, r_{\sim}^{\prime}\right)$, which is the diagonal element of the (fullyinteracting) 2DM. It is encouraging that these two many-electron terms on the RHS of the force-balance eqn. $(5.2){(\underset{\sim}{F}}_{\text {Laplacian }}$ is given directly in terms of the gradient of the Laplacian of the density $\rho(\underset{\sim}{r}))$ are related in the two-electron Hookean atom model discussed in section 2.2 above by the exact result (2.19) and generalizations (albeit approximate) of this to $N$ electrons are of some urgency.

On a related point, future non-local functions of the exchange-correlation energy density $\epsilon_{x c}(\underset{\sim}{r})$ should clearly be constructed with the nuclear-cusp conditions very much in mind, even if, in the end, they can only be embraced semiquantitatively. 


\section{Acknowledgments}

This article could not have come to fruition without invaluable collaboration with Professors A. Holas and I.A. Howard. I am most grateful to them both, not only for the joint contributions in refs.[18] and [17] but also for many most stimulating and informative discussions. Continuous encouragement and help from Dr. P. Senet, and Professors D.J. Klein and V.F. Van Doren is also gratefully acknowledged. 


\section{References}

[1] March, N.H. and Young, W.H. Proc Phys Soc 1958, 72, 182-192.

[2] Herring, C. Phys Rev 1986, A34, 2614-2631.

[3] Herring, C and Chopra, M. Phys Rev 1988, A37, 31-42.

[4] Slater, J.C. Phys Rev 1951, 81, 385-90.

[5] Kohn, W. and Sham, L.J. Phys Rev 1965, 140, A1133-1138.

[6] Wang, L.W. and Teter, M.P. Phys Rev 1992, B45, 13196-13220.

[7] Pearson, M, Smargiassi, E. and Madden, P.A. J Phys Condens Matter 1993, 5, 3221-3240.

[8] Perrot, F. J. Phys Condens Matter 1994, 6, 431-446.

[9] Jesson, B.J., Foley, M. and Madden, P.A. Phys Rev 1997, B55, 4941-4946.

[10] Garciá-González, P., Alvarellos, J.E. and Chacón, E. Phys Rev 1998, B57, 4857=4861.

[11] Holas, A. and March, N.H. Phys Rev 1995, A51, 2040-2048.

[12] Levy, M. and March, N.H. Phys Rev 1997, A55, 1885-1889.

[13] March, N.H. and Young, W.H. Nucl Phys 1959, 12, 237-40.

[14] See, for example, Parr, R.G. and Yang, W. Density Functional Theory of Atoms and Molecules; Oxford University Press, Oxford, 1989.

[15] March, N.H., Senet, P. and Van Doren, V.E. Phys Lett 2000, A270, 88-92.

[16] March, N.H. and White, R.J. J Phys 1972, B5, 466-475.

[17] Howard, I.A. , March, N.H., Senet, P. and Van Doren, V.E. Phys Rev A: in the press.

[18] March, N.H., Howard, I.A., Holas, A., Senet, P. and Van Doren, V.E. Phys Rev A: in press.

[19] Lawes, G.P. and March, N.H. J. Chem Phys 1979, 71, 1007-1009.

[20] Gombás, P. Phys Lett 1969, A28, 585-586.

[21] Goodisman, J. Phys Rev 1970, A1, 1574-1576.

[22] Deb, B.M. and Ghosh, S.K. Int J Quantum Chem 1983, 23, 1-10.

[23] Kestner, N.R. and Sinanog̃lu O. Phys Rev 1962, 128, 2687-2696.

[24] See also Kais, S., Herschbach, D.R., Handy, N.C., Murray, C.W. and Laming, G.J. J Chem Phys 1993, 99, 417-425 and earlier references there. 
[25] March, N.H., Gál, T. and Nagy, Á. Chem Phys Lett 1998, 292, 384-386.

[26] March, N.H., Amovilli, C. and Klein, D.J. Chem Phys Lett 2000, 325, 645-647.

[27] Gál, T., March, N.H. and Nagy, Á. Chem Phys Lett 1999, 305, 429-432.

[28] Hall, G.G., Jones, L.L. and Rees, D. Proc Roy Soc 1965, A283, 194-202.

[29] March, N.H. and Santamaria, R. Phys Rev 1988, A38, 5002-5006.

[30] Dirac, P.A.M. Proc Camb Phil Soc 1930, 26, 376-391.

[31] March, N.H. Phys Rev 1987, A36, 5077-5078.

[32] March, N.H. Phys Rev 1986, A33, 88-89.

[33] Theophilou, A.K. and March N.H. Phys Rev 1986, A34, 3630-3633.

[34] Holas, A. and March, N.H. J Phys A Math Gen 1990, 23, 735-749.

[35] See, for example, Kleinman, L. Phys Rev 1994, B49, 14197-14201.

[36] Harbola, M.K. and Sahni, V. Phys Rev Lett 1989, 62, 489-492.

[37] March, N.H. Phys Rev 2000, B61, 5011-5012.

[38] Kato, T. Commun Pure Appl Math 1957, 10, 151-177;

See also Fraga, S. and Malli, G. Many-electron Systems: properties and interactions (Saunders: Philadelphia), 1968).

[39] Bingel, W.A. Z Naturforsch 1963, 18a, 1249-1258.

[40] Davidson, E.R. Reduced Density Matrices in Quantum Mechanics; Academic Press, New York, 1976.

[41] Harris, J. and Jones, R.O. J Phys 1974, F4, 1170-86.

[42] See also Levy, M., March, N.H. and Handy, N.C. J Chem Phys 1996, 104, 1989-1992. 


\section{Figure Caption}

Figure 1. Shows plot of exchange energy density $\epsilon_{x}(r)$ times $4 \pi r^{2}$ obtained analytically by Howard et al. [17] in the 10-electron bare Coulomb limit system. The large $Z$ necessary is chosen in the figure to be 92 .

Dashed curve shows the Dirac-Slater local density approximation (3.14), calculated with the exact density $\rho(r)$. [Redrawn from Howard et al. [17]]. 